\title{
Prolonged QT dispersion is associated with pediatric syncope
}

\author{
Meki Bilici ${ }^{1}$, Zübeyde Fidanc1-Dedeoğlu², Fikri Demir ${ }^{1}$, Alper Akın¹, Mehmet Türe ${ }^{1}$, \\ Hasan Balık ${ }^{1}$, İlhan Tan², Sabahattin Ertuğrul2 \\ ${ }^{1}$ Division of Pediatric Cardiology, Department of ${ }^{2}$ Pediatrics, Dicle University Faculty of Medicine, Diyarbakır, Turkey. \\ E-mail:drmekibilici@hotmail.com
}

Received: 15th August 2017, Revised: 20th November 2017, Accepted: 17th January 2018

\begin{abstract}
SUMMARY: Bilici M, Fidancı-Dedeoğlu Z, Demir F, Akın A, Türe M, Balık $\mathrm{H}$, Tan $\dot{\mathrm{I}}$, Ertuğrul S. Prolonged QT dispersion is associated with pediatric syncope. Turk J Pediatr 2019; 61: 85-91.

We aimed to find out whether QT dispersion can be used as a diagnostic marker in terms of syncope type, and recurrence risk. Fifty-two patients (28 male, 53.8\%) admitted to the pediatric cardiology clinic with syncope were compared with 50 healthy controls ( 28 male, $56 \%$; mean age: $13.8 \pm 2.3$ years, range: 11-17 years) for QT dispersion (QTd) and other electrocardiographic findings. Gender, age, echocardiography, blood pressure while standing and sitting, electrolyte levels, liver and kidney function tests, and QTd and QTcd (calculated with corrected QT: QTc) in electrocardiography (ECG) of patients were all evaluated. Patients' ages varied between 7-17 years and the mean age at first syncope was $13.9 \pm 2.4$ years. Mean follow-up duration of the patients was $10 \pm 5$ months (range: 5-18 months). Mean number of syncope attacks was $2.8 \pm 2.2$. QTd $(72 \pm 46 \mathrm{~ms}$ vs. $34 \pm 14 \mathrm{~ms})$ and QTcd $(77 \pm 45 \mathrm{~ms}$ vs. $33 \pm 14 \mathrm{~ms}$ ) values of the patients were significantly longer, compared to control group $(p<0.001)$. The age, gender, QTd, and QTcd values did not differ between the subtypes of syncope based on pathogenetic mechanism. Additionally, these parameters did not differ in terms of the number of syncope recurrence and tilt test. Patients' complaints reduced after cardiologic evaluation and advice regarding prevention of syncope. We think that in follow-up period, education and preventive precautions that can be taken in the daily life must be emphasized, and drug treatment can be started in unresponsive cases.
\end{abstract}

Key words: children, syncope, QT dispersion.

Syncope is characterized with sudden loss of consciousness and tonus; it develops by insufficiency of cerebral circulation. ${ }^{1}$ The interruption of cerebral blood flow for 6-8 seconds or $20 \%$ decrease in cerebral oxygenation can cause complete loss of consciousness. Though rarely, without decrease in cerebral blood flow, the decrease in essential matters which are necessary for brain (hypoglycemia etc.) can also cause syncope. Although it is rarely seen, the mortality and morbidity of syncope due to cardiac reasons is high and it has risk of sudden death. ${ }^{2,3}$ For this reason, clinicians have difficulty in persuading patients and unnecessary tests raise cost in follow up of syncope. . $^{2,4}$

QT dispersion (QTd) is a non-invasive parameter which shows the heterogeneity of ventricular repolarization. In 12-lead electrocardiography (ECG), the variability of QT intervals between derivations are defined as QT dispersion. In different study groups, increased QTd has been found to be related to serious arrhythmias and sudden death. ${ }^{5-7}$ In this study, it is aimed to find out QT dispersion value, and to test whether QT dispersion can be used as a diagnostic marker in terms of syncope type, and recurrence risk.

\section{Material and Methods}

\section{Study groups}

This study was conducted at the Pediatric Cardiology Department between October 2014 
Table I. Dispersion Values According to the Syncope Type.

\begin{tabular}{lcccc}
\hline & \multicolumn{4}{c}{ Groups } \\
\cline { 2 - 4 } Features & $\begin{array}{c}\text { Vasovagal syncope } \\
(\mathrm{N}=45)\end{array}$ & $\begin{array}{c}\text { Orthostatic hypotension } \\
(\mathrm{N}=7)\end{array}$ & $\begin{array}{c}\text { Controls } \\
\mathrm{N}=50\end{array}$ & $\mathrm{P}$ \\
\hline Age (years) & $13.8 \pm 2.4$ & $14.7 \pm 2.6$ & $13.8 \pm 2.3$ & $>0.05$ \\
QTd (ms) & $72 \pm 48$ & $74 \pm 38$ & $34 \pm 14$ & $<0.001$ \\
QTCd (ms) & $77 \pm 47$ & $81 \pm 37$ & $33 \pm 14$ & $<0.001$ \\
\hline
\end{tabular}

ms: milliseconds, QTCd: corrected QT dispersion, QTd: QT dispersion

and June 2015. It included 52 patients with syncope and 50 healthy children. The patients with another disease which was thought to cause syncope were not included in the study (congenital heart diseases, rhythm disorders, epilepsy, anemia, drug using etc.). The control group consisted of healthy children who were led to pediatric cardiology with innocent murmur and no pathology was found after examinations.

The study was approved by the Institutional Ethics Committee (20/05/2014-113). The informed approval was taken from parents and patients, as necessary.

\section{Before-after stories of the patients}

Patients and their parents were interviewed for a detailed history. Clinical features before, during and after attacks were recorded; they included number of attacks, coexisting symptoms, position of the patient, presence of prodromal signs, primary complaints before attacks, family history, the way of falling, skin color, loss of consciousness and its duration, and any trauma secondary to syncope. Recurrence of syncope and its features, and adherence to recommendations were recoded, as well.

\section{Physical examination}

A detailed physical examination, including neurological and cardiovascular system was performed. Heart rate, rhythm, and any murmur with its character were recorded. Blood pressure was measured by an appropriate cuff after a rest for 10 minutes in sitting position, then measured again after standing motionless for 10 minutes. A decrease of $20 \mathrm{mmHg}$ or more in systolic pressure and a decrease of $10 \mathrm{mmHg}$ or more in diastolic pressure was defined as orthostatic hypotension.

\section{Laboratory tests}

Complete blood count, liver and kidney function tests, electrolytes (including calcium, phosphorus), thyroid hormone levels, electroencephalography (if necessary), echocardiography, Holter, ECG and tilt test (TT) were performed to elucidate the etiology.

The 12-lead basal ECG of patients were recorded with Nihon Kohden marked device at a speed of $25 \mathrm{~mm} / \mathrm{s}, 10 \mathrm{~mm} / \mathrm{mV}$ amplitude while they were resting in supine position. QT interval was measured as time between QRS complex and end of the $\mathrm{T}$ wave (intersection point of the tangent drawn parallel to the handle which comes down corresponding to the repolarization phase of $\mathrm{T}$ wave with T-P isoelectric line). The derivations whose end point could not be obtained since their $\mathrm{T}$ wave was on isoelectric line were excluded from the study. In the presence of $U$ wave, the end point of QT interval was accepted as the lowest point of the curve between $T$ and $\mathrm{U}$ wave. By measuring three consecutive

Table II. Dispersion Values According to the Number of Syncope.

\begin{tabular}{lccc}
\hline Features & $\begin{array}{c}\text { Number of syncope }<4 \\
(\mathrm{~N}=31)\end{array}$ & $\begin{array}{c}\text { Number of syncope } \\
(\mathrm{N}=21)\end{array}$ & $\mathrm{P}$ \\
\hline Male/female, n (\%) & $19 / 12(61.3 / 38.7)$ & $9 / 12(42.9 / 57.1)$ & $>0.05$ \\
QTd (ms) & $67 \pm 35$ & $80 \pm 60$ & $>0.05$ \\
QTcd (ms) & $75 \pm 93$ & $80 \pm 50$ & $>0.05$ \\
\hline
\end{tabular}

ms: milliseconds, QTCd: corrected QT dispersion, QTd: QT dispersion 
Table III. Dispersion Values According to the Tilt Test Results.

\begin{tabular}{lccc}
\hline Dispersion values & $\begin{array}{c}\text { Positive tilt test } \\
(\mathrm{N}=16)\end{array}$ & $\begin{array}{c}\text { Negative tilt test } \\
(\mathrm{N}=25)\end{array}$ & $\mathrm{P}$ \\
\hline QTd $(\mathrm{ms})$ & $79 \pm 46$ & $74 \pm 50$ & $>0.05$ \\
QTcd $(\mathrm{ms})$ & $83 \pm 45$ & $79 \pm 50$ & $>0.05$ \\
\hline
\end{tabular}

Tit test was performed in 41 patients.

ms: milliseconds, QTCd: corrected QT dispersion, QTd: QT dispersion

beatings in at least nine derivations, QT value was measured in all patients. QTc value was measured with Bazett formula according to the heart rate $(\mathrm{QTC}=$ measured $\mathrm{QT}$ value / $\sqrt{\mathrm{RR}})$. QTd was defined by calculating the difference between minimum and maximum QT values; and QTcd (QTc dispersion) was defined by calculating the difference between minimum and maximum QTc values. All calculations for study and control groups were performed manually by the same person.

\section{Tilt test}

Tilt test (TT) was not applied to patients diagnosed with vasovagal syncope (VVS) through anamnesis and physical examination, while it was applied to patients with questionable diagnosis. Tilt test was applied to patients in the morning, on an empty stomach in a quiet room with the lights slightly dimmed. At the time of the TT none of the patients were using drugs. Hydraulic tilt table on which patients could lean their feet and which could be set up from lie flat position to 80 degrees was used. Patients were tied from their chest, knee and legs with leather belts in case of falling at the time of syncope. Moreover, patients took support from tilt table while in standing position. Patients stayed in supine position for five minutes and basal blood pressure and pulse rate were obtained. Tilt table was set up to 70 degrees and waited for 45 minutes; heart rate and blood pressure were measured at first and fifth minutes. Afterwards, heart beat and blood pressure were recorded every 5 minutes. In the presence any complaint, the measurements were repeated every minute. Test was accepted to be negative in patients with no symptoms after 45 minutes. Syncope was lay in supine position and accepted to be positive.

Patients diagnosed with vasovagal syncope were advised to avoid stimulating factors (crowded and warm places, hunger, dehydration, standing etc.), to be aware of prodromal symptoms and to take appropriate positions, and to increase their fluid and salt intake.

\section{Statistical analysis}

Data were analyzed with SPSS 18.0 (SPSS Inc, Chicago, IL, USA). Whether variants showed a normal distribution or not was assessed by using visual (histogram) and analytical methods (Kolmogrov-Simirnov test). Variants showing normal distribution were displayed as average \pm SD and variants not showing normal distribution were displayed as median (minimum-maximum) values. Student-t test was used to compare independent groups. The statistical analysis of variants designated with rates were made by chi-square test. Results was considered statistically significant when $\mathrm{p}$ value was less than $0.05(\mathrm{p}<0.05)$.

\section{Results}

The study included 52 patients with syncope (28 male, $53.8 \%$; mean age $13.9 \pm 2.4$ years; age range: $7-17$ years) and 50 healthy children (28 male, $50.0 \%$; mean age $13.8 \pm 2.3$ years; age range: $11-17$ years); the groups were comparable for sex and age. Mean follow-up period was $10 \pm 5$ months (range: $6-18$ months). Mean number of syncope were comparable for boys and girls. QT dispersion (72 $\pm 46 \mathrm{~ms}$ vs. $34 \pm 14 \mathrm{~ms})$ and QTc dispersion $(77 \pm 45$ $\mathrm{ms}$ vs. $33 \pm 14 \mathrm{~ms}$ ) were significantly longer in patients, compared to controls $(\mathrm{p}<0.001)$.

Forty-five patients had vasovagal syncope, seven patients had orthostatic hypotension. Patients with orthostatic hypotension did not develop recurrence of syncope during the follow-up period. Fourteen patients in vasovagal syncope group developed recurrence; seven of them had positive tilt test $(p>0.05)$. Among 
Table IV. Dispersion Values and Age Distribution According to Follow-Up Recurrent Syncope Development.

\begin{tabular}{lccc}
\hline Features & $\begin{array}{c}\text { No recurrence } \\
(\mathrm{N}=38)\end{array}$ & $\begin{array}{c}\text { Recurrent syncope } \\
(\mathrm{N}=14)\end{array}$ & $\mathrm{P}$ \\
\hline Age (years) & $13.5 \pm 2.3$ & $15 \pm 2.5$ & 0.06 \\
QTd (ms) & $76 \pm 51$ & $63 \pm 31$ & $>0.05$ \\
QTcd (ms) & $79 \pm 50$ & $72 \pm 32$ & $>0.05$ \\
\hline
\end{tabular}

ms: milliseconds, QTCd: corrected QT dispersion, QTd: QT dispersion

patients with recurrence during the follow-up 9 $(64.3 \%)$ patients had $<4$ syncope and $5(35.7 \%)$ patients had $\geq 4$ syncope. before. Thirty-eight patients had no recurrence during the followup period; $22(57.9 \%)$ patients had a history of $<4$ syncope and $16(42.1 \%)$ patients had a history of $\geq 4$ syncope $(\mathrm{p}>0.05)$.

QTd and QTcd values were comparable when patients were grouped according to syncope type (Table I), number of syncope (Table II), tilt test positivity (Table III), and recurrence of syncope (Table IV).

Holter ECG was performed in 18 patients to rule out rhythm disorders; they were all normal.

\section{Discussion}

Syncope in childhood is very common. Syncope attack is observed at least once in 15 of 100 children in adolescence. ${ }^{8}$ There has not been a consensus on syncope pathology or treatment. However, some imbalances in autonomic nervous system are held responsible for physiopathology. Sometimes without a decrease in cerebral blood flow, decrease in essential matters necessary for brain (hypoglycemia, etc.) can cause syncope. However, we did not detect hypoglycemia in any of the syncope patients. Most common type of syncope is vasovagal syncope. ${ }^{9}$ In a long term study it has been observed that $76 \%$ of the syncope are caused by neural reflex mechanism. ${ }^{10}$ In our study $86.5 \%$ of syncope are vasovagal type.

Especially insufficiency in control mechanism of autonomic cardiovascular system is vital in vasovagal syncope physiopathology. Vasovagal syncope patients do not have the same autonomic stability as healthy people and they are exposed to periodic fluctuations in autonomic nervous system. The imbalance in autonomic nervous system can also be effective on ECG parameters. Especially in recent years this issue has attracted interest and many studies are centered on heart rate variability, QT interval, QTc and dispersion. Findler et al. ${ }^{7}$ showed that QTc is longer in tests of patients with TT. Takahashi et al. ${ }^{11}$ studied on cardiac autonomic functions in 42 patients with type 2 diabetes mellitus and showed extension in vagal dysfunction and QT interval and increase in QTd in presence of sympathic and parasymphatic dysfunction. Furthermore, Akalın et al. ${ }^{12}$ evaluated QTd in cases in which autonomic nervous system disorders are foreground in breath holding in pathophysiology and they determined an increase in QTd when compared to the control group. In our study QTc and QTd durations of patients with vasovagal syncope were quite longer in comparison with the control group. Since autonomic nervous system imbalance which are blamed for physiopathology of orthostatic tolerance disorder have effects on cardiac conduction system, we think it plays role in QTd and QTcd prolongation in patients with syncope.

In children with vasovagal syncope the rate of TT positivity without medication is between $10-44 \% .13-15$ This rate can rise up to $80 \%$ in TT with medication ${ }^{16}$. In pediatric studies including control groups the TT sensitivity without medication is between $43-49 \%$ and specificity is between $93-100 \% .{ }^{13,17,18}$ In TT in which isoproterenol is used as a stimulant, sensitivity rises up to $77 \%$ but specificity drops down to $87 \%$. In various researches it has been claimed that using stimulants such as isoproterenol in TT reduces specificity and causes errors and complexity in results. ${ }^{19-21}$ American College of Cardiology reported that TT without using stimulant medicine is more useful than other protocols in symptomatic patients and in differential diagnosis of asymptomatic controls. ${ }^{21}$ Therefore, stimulant 
medicine wasn't used in our study and test results in $39 \%$ of patients were positive as compatible with literature.

Kula et al. ${ }^{22}$ determined a significant increase in QTC dispersion in the early morning and in late hours of night. This situation is related to upgrade symptomatic activities in these hours and accepted as the reason for the increase in syncope attacks at same hours. Lacoviello et al. ${ }^{23}$ determined as a result of a study applied to 190 adult patients that among the factors predicting the risk of VVS relapse, number of previous syncope attacks along with suppressed baroreceptor reflex during TT are important variables in female gender. However, in our study when TT positive group was compared to TT negative group a significant difference between QTd and QTcd were not determined. Koukam et al. ${ }^{24}$ applied TT to 101 children and observed no difference in female and male gender in terms of positive and negative TT results. In our study we have not found a relation between TT results and gender.

Although syncope is common in children and adolescents and so much research is carried out on vasovagal syncope in children, there are not enough studies on predicting recurrence factors. It is still unclear to estimate the recurrence risk of syncope in children having experienced vasovagal syncope. After routine use of TT to diagnose syncope, some studies have been conducted about the role of TT results and the variables in some parameters during the test in recurrence estimate. While history is enough for diagnosis in some of syncope attacks, in others TT is needed. Test provides an orthostatic environment facilitating formation of vasovagal syncope and it has high diagnostic value to evaluate the syncope which is presented in patients not having structural heart disease. Some research on unexplained syncope showed that TT implies limited contribution to diagnosis of $40-70 \%$ of the patients. Another study on unexplained syncope in 201 patients who does not represent any other diseases showed that the reduced baroreflex control of the heart plays an independent and increasing role in recurrent syncope. ${ }^{25}$ Also, we can conclude that TT shouldn't be requested for every patient routinely as we have $11(21.2 \%)$ patients diagnosed by only detailed history and physical examination and they have not presented syncope again in follow up period.

Our data shows that TT application does not have therapeutic effect on recurrent syncope. In a study, it is claimed that TT positive shouldn't be accepted as an inappropriate prognostic marker, but frequency of recent attacks is a more valuable prediction. ${ }^{26}$ It is remarked that patients with TT positive and 6 or more syncope attacks have more than $50 \%$ risk of recurrence in two years period. ${ }^{22}$ Koukam et al. ${ }^{24}$ remarked the syncope recurrence rate as $32 \%$ in children and adolescence and they reported that in TT positive and in TT negative patients there is no difference in syncope recurrence. Salim et al. ${ }^{27}$ pointed out that the recurrent syncope is higher in TT positive patients. In our study we have not found a significant relation between TT positive and syncope recurrence.

In long term placebo-controlled studies, there is no significant difference between medicine treatment and placebo. ${ }^{28}$ Koukam et al. ${ }^{24}$ followed up 67 children with vasovagal syncope by giving medicine treatment to 43 of them while the other 24 children didn't get a treatment. At the end of 4 years follow up they haven't found a difference with regard to syncope recurrence between the treated group and the other one. ${ }^{25}$ Grimm et al. ${ }^{29}$ followed up 80 patients for $23 \pm 8$ months and in $90 \%$ of patients who had only one syncope attack independently from TT results (without medical treatment) no syncope recurrence was observed. In another study, in half of patients who presented two or more previous syncope attacks syncope recurrence were observed and these patients are thought to be suitable candidates for pharmacologic treatment.

There were a number of limitations in this study. First, the present study is a retrospective analysis and therefore limited by the potential for selection bias. The second limitation was the relatively small sample size and the third was the relatively short duration of follow-up. The study is also a single center study and further investigations would be needed to evaluate the generalizability of these findings.

In this study, we observed that QT dispersion and QTC dispersion significantly lengthen in children with syncope but these findings do not have significant effect on syncope recurrence. Tilt test results also have no effect on syncope 
recurrence. Patients' complaints reduced after pediatric cardiologic evaluation and clinicians advices regarding prevention of syncope. In treatment, we think that patient education, preventive precautions in daily life should be emphasized more, fluid intake should be increased and in necessary cases medicine treatment should be started.

\section{REFERENCES}

1. Koca S, Paç AF, Ece İ, Bağrul D, Çay S. Cardiac nodal and cardiac autonomic functions in children with vasovagal syncope. Turk J Pediatr 2016; 58: 498-502.

2. Zhang Q, Du J, Wang C, Du Z, Wang L. Tang C. The diagnostic protocol in children and adolescents with syncope: A multi-centre prospective study. Acta Paediatr 2009; 98: 879-884.

3. DiMario FJ Jr, Wheeler Castillo CS. Clinical categorization of childhood syncope. J Child Neurol 2011; 26: 548-551.

4. Steinberg LA, Knilans TK. Syncope in children: Diagnostic tests have high cost and low yield. J Pediatr 2005; 146: 355-358.

5. Strickberger SA, Benson DW, Biaggioni I, et al; American Heart Association Councils on Clinical Cardiology, Cardiovascular Nursing, Cardiovascular Disease in the Young, and Stroke; Quality of Care and Outcomes Research Interdisciplinary Working Group; American College of Cardiology Foundation; Heart Rhythm Society; American Autonomic Society. AHA/ACCF Scientific statement on the evaluation of syncope: from the American Heart Association Councils on Clinical Cardiology, Cardiovascular Nursing, Cardiovascular Disease in the Young, and Stroke, and the Quality of Care and Outcomes Research Interdisciplinary Working Group; and the American College of Cardiology Foundation: in collaboration with the Heart Rhythm Society: endorsed by the American Autonomic Society.. Circulation 2006; 113: 316-327.

6. Cetinkaya M, Semizel E, Bostan O, Cil E. Risk of vasovagal syncope and cardiac arrhythmias in children with mitral valve prolapse. Acta Cardiol 2008; 63: 395-398.

7. Findler M, Birger A, Diamant S, Viskin S. Effects of head-up tilt-table test on the QT interval. Ann Noninvasive Electrocardiol 2010; 15: 245-249.

8. Tanrıverdi Yılmaz S, Binnetoğlu K, Babaoğlu K, Altun G. Predictors of vasovagal syncope recurrence in children and adolescents and value of head-up tilt table test. Anadolu Kardiyol Derg 2013; 13: 688-694.

9. Manolis AS, Linzer M, Salem D, Estes III NAM. Syncope: Current diagnostic evaluation and management. Ann Intern Med 1990; 112: 850-863.

10. Benditt DG, Ferguson DW, Grubb BP, et al. Tilt table testing for assessing syncope. American College of Cardiology. J Am Coll Cardiol 1996; 28: 263-275.

11. Takahashi N, Nakagawa M, Saikawa T, et al. Regulation of QT indices mediated by autonomic nervous function in patients with type 2 diabetes. Int J Cardiol 2004; 96: $375-379$
12. Akalın F, Turan S, Güran T, Ayabakan C, Yılmaz Y. Increased QT dispersion in breath-holding spells. Acta Paediatr 2004; 93: 770-774.

13. Ross BA, Hughes S, Anderson E, Gillette PC. Abnormal responses to orthostatic testing in children and adolescents with recurrent unexplained syncope. Am Heart J 1991; 122 (3 Pt 1): 748-754.

14. Grubb BP, Temesy-Armos P, Moore J, Wolfe D, Hahn $\mathrm{H}$, Elliott $\mathrm{L}$. The use of head-upright tilt table testing in the evaluation and management of syncope in children and adolescents. Pacing Clin Electrophysiol 1992; 15: 742-748.

15. Pollini I, Favilli S, De Simone L, Romanelli AM, Manetti A. Syncope at paediatric ages: Evaluation with head up tilt. Cardiologia 1998; 43: 499-503.

16. Pongiglione G, Fish FA, Strasburger JF, Benson DW Jr. Heart rate and blood pressure response to upright tilt in young patients with unexplained syncope. J Am Coll Cardiol 1990; 16: 165-170.

17. Lewis DA, Zlotocha J, Henke L, Dhala A. Specificity of head-up tilt testing in adolescents: Effects of various degrees of tilt challenge in normal control subjects. J Am Coll Cardiol 1997; 30: 1057-1060.

18. Lerman-Sagie T, Rechavia E, Strasberg B, Sagie A, Blieden L, Mimouni M. Head-up tilt for the evaluation of syncope of unknown origin in children. J Pediatr 1991; 118: 676-679.

19. Kapoor WN, Brant N. Evaluation of syncope by upright tilt testing with isoproterenol. A nonspecifc test. Ann Intem Med 1992; 116: 358-363.

20. Alehan D, Lenk M, Ozme S, Celiker A, Ozer S. Comparison of sensitivity and specificity of tilt protocols with and without isoproterenol in children with unexplained syncope. Pacing Clin Electrophysiol 1997; 20: 1769-1776.

21. Benditt DG, Ferguson DW, Grubb BP, et al. Tilt table testing for assessing syncope. American College of Cardiology. J Am Coll Cardiol 1996; 28: 263-275.

22. Kula S, Olgunturk R, Tunaoglu FS, Canter B. Circadian variation of QTc dispersion in children with vasovagal syncope. Int J Cardiol 2004; 97: 407-410.

23. Iacoviello $\mathrm{M}$, Forleo $\mathrm{C}$, Guida $\mathrm{P}$, et al. Independent role of reduced arterial baroreflex sensitivity during head-up tilt testing in predicting vasovagal syncope recurrence. Europace 2010; 12: 1149-1155.

24. Koukam C, Vaksmann G, Pachy E, Lacroix D, Rey C, Kacet S. Long-term follow-up of children and adolescents with syncope; predictor of syncope recurrence. Eur Heart J 2001; 22: 1618-1625.

25. Díaz FJ, Tercedor L, Moreno E, et al. Vasovagal syncope in pediatric patients: A medium-term follow-up analysis Res Esp Cardiol 2002; 55: 487-492.

26. Zabel M, Woosley RL, Franz MR. Is dispersion of ventricular repolarization rate dependent? Pacing Clin Electrophysiol 1997; 20: 2405-2411.

27. Salim MA, Ware LE, Barnard M, Alpert BS, DiSessa TG. Syncope recurrence in children: Relation to tilttest results. Pediatrics 1998; 102: 924-926. 
28. Biffi M, Boriani G, Bronzetti G, Frabetti L, Picchio FM, Branzi A. Neurocardiogenic syncope in selected pediatric patients-natural history during long-term follow-up and effect of prophylactic pharmacological therapy. Cardiovasc Drugs Ther 2001; 15: 161-167.
29. Grimm W, Degenhardt M, Hoffmann J, Menz W, Wirths A, Maisch B. Syncope recurrence can better be predicted by history than by head-up tilt testing in untreated patients with suspected neurally mediated syncope. Eur Heart J 1997; 18: 1465-1469. 